\title{
DIFFERENTIAL DIAGNOSIS OF WHOLE-BODY VIBRATION RELATED DISORDERS
}

\author{
Dr. med. ECKARDT JOHANNING MD, MSc. \\ Occupational and Environmental Life Science, Albany, N.Y.
}

\section{Introduction}

Occupational whole-body vibration (WBV) exposure can lead to multiple, often nonspecific health complaints and to physical disorders in vehicle operators. Primarily musculoskeletal or neurological disorders of the spine are generally recognized adverse health outcomes of intense and prolonged exposures due to strong epidemiological evidence. In some countries these types of "mechanical" repetitive back injuries are compensable if certain criteria are met. ${ }^{12}$ Less frequently organ damage has been reported including the prostate, the intestinal organs (stomach, bladder and kidneys), female reproductive organs, peripheral veins, hemorrhoids, or the cochlear vestibular system. ${ }^{34}$ The medical diagnosis, treatment and prevention of occupational low back disorders has been reviewed in a clinical consensus protocol. ${ }^{5}$ A prototype health surveillance scheme for WBV has been proposed. ${ }^{6}$ However, many physicians and occupational safety and health experts seem not to be familiar with the wide spectrum of health complaints and the proper diagnostic evaluation of WBV-exposed patients. ${ }^{7}$ The aim of this study is to review the current medical knowledge and differential diagnostic evaluation methodology.

\section{Methods}

Review of peer-reviewed clinical reports and scientific journal publications of wholebody vibration related health effects and the medical differential diagnostic evaluation protocols and recommendations. Review of the evidence and differential diagnosis.

\section{Results}

There is general recognition that the diagnostic approach for the medical evaluation of cases with suspected vibration-induced disorders should include a comprehensive work history, a list of hobbies that may include vibration exposure, a complete medical -, family- and social history, review of systems (smoking habits), medications, prior injury and trauma, followed by a complete physical examination. In addition, some special diagnostic laboratory and imaging studies may be indicated to rule out "red flag" conditions. WBV is frequently associated with symptoms such as acute or chronic low back pain, peripheral neuropathy and progressive degenerative changes of the spine, including lumbar inter-vertebral disc disorders. Typically, significant symptoms and signs are severe and chronic pain, paresthesia and neurological dysfunction. To a lesser degree WBV exposure may also lead to neck-shoulder problems, digestive disorders, circulatory disorders, auditory effects, and reproductive problems. The health 
complaints are typically discomfort, pain or organ related dysfunction. The differential diagnosis includes pathologies principally related to acute trauma (fractures), infections (i.e., tuberculosis), cancer, systemic and chronic diseases (i.e., osteoporosis, auto-immune diseases, inflammatory rheumatologic problems, metabolic diseases (diabetes, thyroid), bone, prostate, renal or psychiatric disorders. Diagnostic tests include blood and urine laboratory studies, radiological imaging and electrophysiological studies. Symptoms, pathology, and radiologic appearances may be only weakly correlated. The prevalence of a prolapsed intervertebral disk among persons with low back pain in primary care is estimated to be 1 to 3 percent. About 4 percent of patients with low back pain in primary care settings have a compression fracture, and about 1 percent have a tumor. Inflammatory rheumatologic conditions (ankylosing spondylitis) and spinal infections are typically less common. The causal diagnosis is based on the medical findings, the differential diagnostic methodology and supportive work exposure data (WBV vibration measurements according to national or international (i.e., ISO 2631-1, ACGIH) guidelines and supportive literature.

\section{Discussion}

A proper WBV-related injury diagnosis includes a critical review of the work history, exposure data assessment and the clinical differential diagnostic evaluation. Many health care providers receive little or no training in occupational medicine and recognition of WBV related injuries. The aims of improved WBV health surveillance are to assess health status and improve the diagnosis of vibration-induced disorders at an early stage, to inform the workers on the potential risk associated with vibration exposure, to give preventive advice to employers and employees. Customized occupational and clinical health questionnaires and examination protocols may be useful in the systematic medical monitoring of WBV exposed workers and should me more utilized in the medical evaluation.

\section{Reference List}

1. C. T. Hulshof et al. The fate of Mrs. Robinson: Criteria for recognition of whole-body vibration injury as an occupational disease, 253 J SOUND VIBRATION.185, 185-194 (2002).

2. K. TESCHKE et al. Whole body vibration and back disorders among motor vehicle drivers and heavy equipment operators - A review of the Scientific evidence. (1999). http://www.cher.ubc.ca/PDFs/WBV_Report.pdf

3. H. Seidel \& R. Heide,. Long-term effects of whole-body vibration: a critical survey of the literature, 58 INT.ARCH.OCCUP.ENVIRON.HEALTH.1, 1-26 (1986).

4. M. Bovenzi,. Criteria for case definitions for upper limb and lower back disorders caused by mechanical vibration, 98 MED.LAV.98, 98-110 (2007).

5. E. Johanning,. Evaluation and management of occupational low back disorders 2, 37 AM.J.IND.MED.94, 94-111 (2000).

6. M. Bovenzi \& C. T. Hulshof. Risk of occupational vibration exposure (VIBRISKS) ANNEX 21 TO FINAL TECHNICAL REPORT. (2007). http://www.vibrisks.soton.ac.uk/body_reports.htm

7. A. R. Last \& K. Hulbert,. Chronic low back pain: evaluation and management, 79 AM.FAM.PHYSICIAN.1067, 1067-1074 (2009). 